\title{
Editorial: Biologically Inspired Computing and Networking
}

\author{
Yifan Chen ${ }^{1} \cdot$ Tadashi Nakano ${ }^{2} \cdot$ Lin Lin $^{3} \cdot$ Weisi Guo ${ }^{4} \cdot$ Mohammad U. Mahfuz $^{5}$ \\ Published online: 3 May 2021 \\ (C) The Author(s), under exclusive licence to Springer Science+Business Media, LLC, part of Springer Nature 2021
}

\section{Editorial:}

Biologically inspired computing and networking represents an emerging research field that seeks the understanding of key principles, processes and mechanisms in biological systems and leverages the understanding to develop novel computing and networking mechanisms. This special issue features five selected recent research efforts in biologically inspired computing and networking.

The first paper entitled "Distributed Power Controller of Massive Wireless Body Area Networks Based on Deep Reinforcement Learning", authored by P. He et al., addresses the energy saving issue of massive wireless body area networks (WBAN). A distributed power controller is developed based on deep Q-learning algorithm to mitigate the affection of inter-network interference. Simulation results demonstrate the significant improvement of proposed scheme in terms of energy efficiency.

In order to solve the problem of low classification accuracy of motor imagery caused by the differences between individual electroencephalogram (EEG) signals, the second paper, "A Personalized Feature Extraction and Classification Method for Motor Imagery Recognition" authored by J.G. Wang et al., proposes a personalized feature extraction method based on filter bank and elastic net and a personalized channel selection based on Deep Belief Network (DBN). According to individual differences, feature vectors and channels containing more classification information can be flexibly selected, avoiding manual adjustment of a specific frequency range for feature extraction and inputting all channels in traditional common spatial pattern (CSP) algorithm.

The third paper, entitled "Real-Time Monocular Obstacle Detection Based on Horizon Line and Saliency Estimation for Unmanned Surface Vehicle" authored by Z. Rui et al., presents a real-time monocular obstacle detection method based on horizon line and saliency estimation for USVs. Specifically, a novel semantic segmentation method based on Gaussian mixture model (GMM) and Markov random field (MRF) is designed to detect the horizon line, which outperforms existing methods based on edge or line features. Inspired by human visual attention mechanisms, an efficient saliency detection method based on background prior and contrast prior is presented to detect obstacles below the estimated horizon line. To reduce false positives caused by sun glitter, waves, and foam, the continuity of the adjacent frames is employed to filter the detected obstacles.

Because of the development of the IoT technologies, various industries have applied a large number of IoT devices. However, the Internet of Things usually does not have a sufficient computing power, so the simple data encryption standard (DES) with lower security is usually used as the main encryption method. The fourth paper, "A High Security Symmetric Key Generation by using Genetic Algorithm based

\author{
Tadashi Nakano \\ tadasi.nakano@fbs.osaka-u.ac.jp \\ Yifan Chen \\ yifan.chen@uestc.edu.cn \\ Lin Lin \\ fxlinlin@ tongji.edu.cn \\ Weisi Guo \\ weisi.guo@warwick.ac.uk \\ Mohammad U. Mahfuz \\ mahfuzm@uwgb.edu
}

1 University of Electronic Science and Technology of China, 2006 Xiyuan Blvd., West High-Tech District, Chengdu, Sichuan, China

2 Graduate School of Engineering, Osaka City University, 3 Chome-3-138 Sugimoto, Sumiyoshi Ward, Osaka 558-8585, Japan

3 College of Electronics and Information Engineering, Tongji University, 1238 Gonghexin Rd, Zhongshan North Road, Zha Bei Qu, Shanghai, China

4 Human Machine Intelligence, Cranfield University, College Rd, Cranfield, Wharley End, Bedford MK43 0AL, UK

5 Richard J. Resch School of Engineering, University of Wisconsin-Green Bay, 2420 Nicolet Dr, Green Bay, WI 54311, USA 
on A Novel Similarity Model" authored by M.-Y. Tsai, proposes a DES encryption based on genetic algorithm with a novel fitness function, which greatly reduce the weakness of the original DES encountering weak keys.

Finally, the paper "Developing an Intelligent Agricultural System based on Long Short-Term Memory" authored by H.$\mathrm{T}$. Wu develops an intelligent agriculture system based on Long Short-Term Memory (LSTM). The system develops an Internet of Things (IoT) to monitor the environmental conditions of soil, sunlight, and temperature; additionally, the research combines the information from the Central Weather Bureau for predicting the timing for watering and notifying farmers about the suggested amount of pesticides and fertilizers.

These five research papers together highlight recent advances in the emerging interdisciplinary field of biologically inspired networking. Although the field of biologically inspired networking has a long history, further research is needed for practical application and development. We hope that the special issue stimulates further research in the field. To conclude the editorial remarks, we thank the Editor-in-Chief, Dr. Imrich Chlamtac for helping us organize the special issue. We also thank all authors and reviewers who contributed to this special issue.

Publisher's note Springer Nature remains neutral with regard to jurisdictional claims in published maps and institutional affiliations.

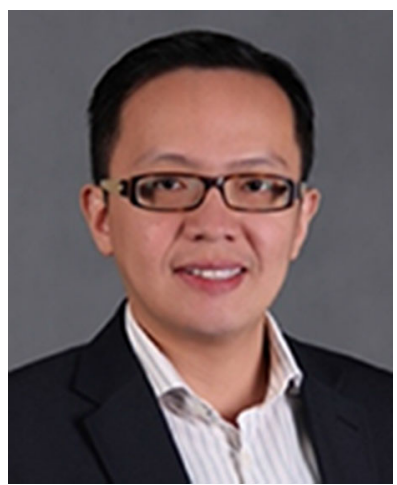

Yifan Chen received the B.Eng. (Hons I) and Ph.D. degrees in electrical and electronic engineering from Nanyang Technological University (NTU), Singapore, in 2002 and 2006, respectively. He has held various academic and leadership positions in the universities in China, New Zealand, U.K., and Singapore. His current research interests include computational nanobiosensing, ICTinspired nanobiomedicine, and electromagnetic medical imaging and sensing for low-cost and lightweight healthcare

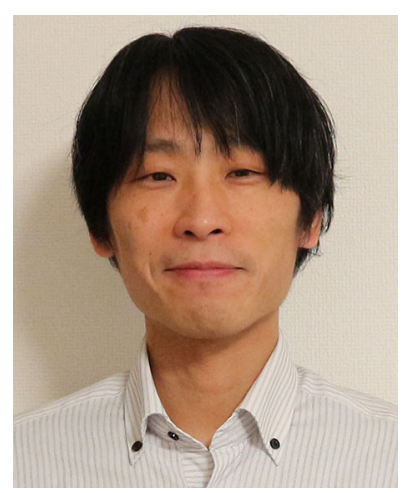

Tadashi Nakano is a Professor of the Graduate School of Engineering, Osaka City University, and a visiting Professor of the Graduate School of Frontier Bioscience, Osaka University. He has been engaged in research at the intersection of computer science and biology, including design, implementation, and evaluation of molecular communication systems, synthetic biological systems, and biologically inspired systems.

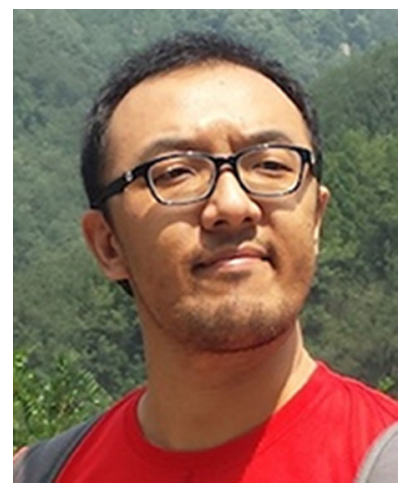

Lin Lin received the B.S. and M.S. degrees in electrical engineering from Tianjin University, Shanghai, China, in 2004 and 2007, respectively, and the $\mathrm{Ph} . \mathrm{D}$. degree from Nanyang Technological University, Singapore, in 2012. He is currently an Associate Professor with the College of Electronics and Information Engineering, Tongji University. His research interests include molecular communication, nanonetworks, and body sensor networks

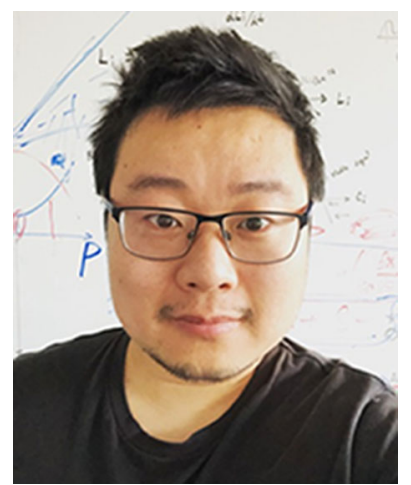

Weisi Guo (S07, M11, SM17) received his MEng, MA, and Ph.D. degrees from the University of Cambridge, UK. He is Chair Professor of Human Machine Intelligence at Cranfield University. He has published over 180 papers and is PI on a number of molecular communication research grants. His research has won him several international awards (IET Innovation 15, Bell Labs Prize Finalist 14, 16, and 19). He was a Turing Fellow at the Alan Turing Institute and Fellow of Royal Statistical Society 


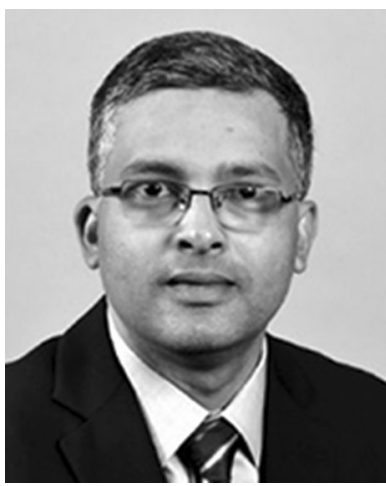

Mohammad U. Mahfuz (M'15) earned his $\mathrm{Ph} . \mathrm{D}$. degree in electrical and computer engineering from the University of Ottawa in Canada in December 2014. Prior to that, he completed his M.Sc. degree in Geomatics Engineering from the University of Calgary, Canada, in September 2008, M.Engg. degree in Telecommunications from the Asian Institute of Technology (AIT), Thailand, in May 2005, and B.Sc. degree in Electrical and Electronic Engineering from the Bangladesh University of Engineering and Technology (BUET) in Dhaka, Bangladesh, in April 2002. Dr. Mahfuz is currently an associate professor of Electrical
Engineering at the Richard J. Resch School of Engineering at the University of Wisconsin-Green Bay, Wisconsin, USA. His research interests include nanoscale communication systems and nanonetworks, ultrawideband wireless communication systems, and renewable energy and information and communication technology (ICT) for sustainable development. 